\title{
Proceso de armonización de excombatientes pertenecientes al Pueblo Indígena Nasa: una oportunidad para la construcción de paz territorial y buen \\ vivir $^{*}$
}

\section{Harmonization process of ex-combatants from Nasa Indigenous People: an opportunity for the construction of territorial peace and good living}

María Vanessa López León ${ }^{* *}$

\section{Resumen}

En el año 2016, Colombia presenció un hito que marcó su historia: la firma de los acuerdos de paz entre el gobierno Santos y las FARC-EP; en él se acordaron metas y estrategias para la reincorporación de excombatientes pertenecientes a comunidades indígenas. La implementación de estas metas y estrategias atraviesa un momento crítico, donde su futuro es incierto. Sin embargo, desde tiempo atrás se han conocido experiencias e iniciativas significativas por parte de algunas comunidades para llevar a cabo la reincorporación y el retorno de excombatientes pertenecientes a estas. El presente artículo tiene como objetivo desarrollar un breve

Artículo de Investigación.

** Politóloga, Universidad de San Buenaventura, sede Bogotá; graduada con trabajo de grado meritorio; activista y defensora de los Derechos Humanos. 
análisis de cómo el programa de armonización (reincorporación) de excombatientes pertenecientes a la comunidad indígena nasa -el programa Recomponer un camino de vuelta a casa- logra configurarse como una oportunidad para la construcción de paz territorial y el buen vivir.

\section{Palabras claves}

Armonización, Acuerdos de Paz, los nasa, paz territorial, buen vivir.

\section{Abstract}

In 2016, Colombia witnessed a historical event: the signing of the peace agreements between the Santos government and the FARC-EP; In it, goals and strategies for the reincorporation of excombatants belonging to indigenous communities were agreed. The implementation of these goals and strategies is going through a critical moment where its future is uncertain. However, some experiences and initiatives have been known for some time by some communities to carry out the reincorporation and return of former combatants belonging to them. This article aims to develop a brief analysis of how the harmonization program (reincorporation) of excombatants belonging to the Nasa community - the Recompose a way back home program - manages to be configured as an opportunity for the construction of territorial peace and Good Living.

\section{Keywords}

Harmonization, Peace agreements, Nasa community, territorial peace, Good living. 


\section{Introducción}

A finales del año 2016 se dio por concluida la etapa de diálogo y negociación entre delegados del Gobierno Nacional de Colombia, en cabeza del presidente de la República Juan Manuel Santos y la antigua guerrilla de las Fuerzas Armadas Revolucionarias de Colombia - Ejército del Pueblo (FARC-EP). Dichos diálogos y negociaciones pretendían darle fin a un conflicto armado que se había postergado por más de 50 años a lo largo y ancho del territorio nacional. Con el cierre de esta etapa se le dio fin a un largo camino, que empezaba en el año 2012 cuando se instauró inicialmente el primer Encuentro Exploratorio y, posteriormente, la Mesa de Conversaciones, que concluyó en noviembre del año 2016 con la firma del Acuerdo Final para la Construcción de una Paz Estable y Duradera (en adelante Acuerdos de Paz).

Hoy, cumplidos cuatro años desde la firma de estos Acuerdos de Paz en el 2016, se hace relevante revisar el estado en el que se encuentran todas las metas y los alcances que se imprimieron sobre el mismo y que con orgullo anunciaban como un hecho histórico cargado de un enorme potencial transformador, que, si bien pretendía terminar la contienda político-militar entre las FARC-EP y el Gobierno Nacional, propendió también por la planteamiento de propuestas y reformas que facilitarían la construcción de soluciones estructurales, lo cual paulatinamente desmontarían las condiciones y los factores, por los cuales el conflicto persiste y se ha reproducido durante años; dichas propuestas y reformas se encuentran a lo largo de los 6 puntos que conforman los Acuerdos de Paz. Al respecto, el docente universitario Jairo Estrada mencionó en su libro El acuerdo de paz en Colombia: entre la perfidia y la potencia transformadora que:

La dimensión de los efectos políticos y culturales se sitúa más bien en perspectiva histórica, posee alcances de nivel sistémico y estructural e indaga acerca de la capacidad transformadora que comporta, o no, la materialización 
del Acuerdo de paz sobre la organización del poder y la dominación de clase $y$, con ello, también sobre la tendencia del proceso de acumulación capitalista. (Estrada, 2019, p. 23)

En el marco de los 6 puntos que conforman los Acuerdos de Paz se acordó la construcción de un programa diferenciado y especial que permitiera la reincorporación de excombatientes pertenecientes a pueblos indígenas, esto con el objetivo de construir una ruta que les posibilitara a estas personas, retornar a sus comunidades (Acuerdo final para la terminación del conflicto y la construcción de una paz estable y duradera, 2016). Cabe resaltar que desde varias colectividades, y en especial en el Pueblo Nasa, ya se contaban con experiencias propias que habían sido construidas años atrás, esto con la intención de permitirle a los miembros de sus comunidades el retorno, logrando recuperar su armonía con el territorio y el tejido social, haciendo que se constituya como un proceso desde una perspectiva holística, incluso abarcando el componente espiritual. La diferencia sustancial entre el concepto de "reincorporación", establecido desde la perspectiva institucional, y el proceso de "armonización", planteado por las comunidades indígenas, cobra prevalencia en esta construcción, puesto que es fundamental para acercarse a la experiencia ya mencionada, la cual fue construida en el año 2007 por la comunidad nasa, y se denominó "Recomponer un camino de vuelta a casa”.

El presente artículo tiene como objetivo desarrollar un análisis sucinto, bajo el método cualitativo, de cómo el programa de armonización de excombatientes pertenecientes a la comunidad indígena nasa -el programa "Recomponer un camino de Vuelta a Casa"- logra configurarse como una oportunidad para la construcción de paz territorial y el Buen Vivir. Para esto se desarrolló una aproximación al proceso de paz, dando un recorrido por documentos imperantes que evidencian, desde lo pactado en los acuerdos de paz, la ruta para el proceso de reincorporación de excombatientes indígenas. Posteriormente, se buscó realizar 
una aproximación académica a los conceptos de 'Buen Vivir' y 'paz territorial', para después tejer un diálogo entre la perspectiva institucional y la perspectiva de las comunidades sobre estos conceptos, esto con el objetivo de evidenciar que, si bien los acuerdos poseen un potencial transformador desde su implementación e incluso desde lo pactado, hay una dificultad dentro del proceso de armonización por establecer escenarios de paz en los territorios que respeten la autonomía, experiencias vitales y formas de vida de las comunidades que los habitan.

A su vez, se pretende establecer que, por el contrario, hay una intención por absorber las dinámicas comunitarias propias de la comunidad indígena nasa, rompiendo y acercando sus procesos y plan de vida a las lógicas institucionales del Estado, intensión que se encuentra en una constante tensión con las comunidades que pugnan por desarrollar un proceso autónomo de armonización, coherente con sus saberes, formas de vida, apuestas, prácticas y disputas.

\section{Contexto}

Los nasa son una comunidad indígena que habita milenariamente en los departamentos del Cauca, algunas regiones del Huila, Valle del Cauca y Putumayo. Según fuentes del DANE, en el censo del año 2018 se reportó que 243176 personas se autoreconocieron como pertenecientes al pueblo nasa, convirtiéndose en el cuarto pueblo más numeroso del país. Para el censo del 2005, el pueblo Nasa se concentraba

(...) en el departamento del Cauca, en donde habita el 88,6\% de la población (164.973 personas). Le sigue Valle del Cauca con el 3,8\% (7005 personas) y Putumayo con el 1,7\% (3190 personas). Estos tres departamentos concentran el $94,1 \%$ poblacional de este pueblo. Los Nasa representan el $13,4 \%$ de la población indígena de Colombia. (Procuraduria General de la Nación, 2019, p. 3). 
Estos territorios habitados por los nasa se han constituido como zonas de alta importancia para múltiples estructuras armadas legales e ilegales, en tanto son estratégicos como corredores o como centros de acopio, distribución, puntos de extracción y comercialización (minería, monocultivos, cultivos de uso ilícito) o de dominio militar, razón por la cual históricamente han sufrido las consecuencias directas del conflicto armado y se ha convertido en un territorio en disputa por múltiples actores, que a través de la violencia han pretendido continuar con el saqueo y la apropiación del territorio indígena. Frente a esta situación, los nasa han encontrado múltiples formas de resistencia pacífica, encaminadas a la preservación de sus raíces y la defensa de su territorio y la comunidad como fuente vital de vida.

Hay varios elementos que se deben tener en cuenta para entender esta reproducción del conflicto sobre todo el departamento del Cauca -donde habita la mayoría del pueblo Nasa-. Entre los más relevantes, parafraseando a Alejandra Miller (2020) en el informe presentado a la Comisión de la Verdad, se encuentran las condiciones de desigualdad, pobreza, exclusión, corrupción, intereses de acumulación en manos de empresas transnacionales, el incumplimiento sistemático de más de 2000 acuerdos firmados entre las comunidades y distintos gobiernos y, actualmente, el incumplimiento de los acuerdos de paz.

Frente a este panorama, el programa "Recomponer un camino de vuelta a casa" es una de muchas herramientas para la resistencia pacífica que la nasa han configurado en los últimos años. Es una propuesta que en el año 2007 la Asociación de Cabildos Indígenas del Norte del Cauca (ACIN) y la comunidad nasa, de la mano del Instituto Colombiano de Bienestar Familiar (ICBF), construyeron para solucionar una problemática a la cual esta comunidad se ha enfrentado durante años: la vinculación y reclutamiento de niños, niñas y adolescentes indígenas por parte de grupos armados, en especial las FARC-EP. Esto se hizo teniendo en cuenta que, en el censo 
socioeconómico realizado por la Universidad Nacional de Colombia en el año 2017 a los excombatientes, evidenció que, de las 10015 personas censadas, el $18 \%$ pertenecía a algún pueblo indígena (Universidad Nacional de Colombia, 2017).

El programa puede entenderse como un esfuerzo por la construcción de una ruta de reincorporación con enfoque étnico dirigido a estos jóvenes (y también a adultos si se hace de forma voluntaria) indígenas, que por diferentes razones entraron a formar parte de las filas de las extintas FARC-EP. Lo anterior establece un proceso comunitario, mediante el cual se pretende orientar a los jóvenes y los adultos para que se acojan y retornen a su comunidad y restablezcan la armonía con el territorio. A través de este programa y su carácter espiritual se busca la recuperación del pensamiento nasa y "armonizar a la persona para que el corazón y el pensamiento se abran” (Verdad Abierta, 2015).

También cabe resaltar que los jóvenes y adultos que se encuentran dentro del programa deben cumplir con compromisos concretos, como lo son terminar sus estudios para posteriormente vincularse a talleres de formación, proyectos productivos, e incluso, proyectos que permitan el fortalecimiento de la vida política y organizativa de la comunidad, por ejemplo, la Guardia Indígena como componente fundamental en la defensa del territorio.

Es fundamental también reconocer que iniciativas de este tipo pueden ser estrategias que desde la comunidad le apuestan a la construcción de escenarios territoriales de paz y la conformación de buenos vivires, enraizados profundamente en sus costumbres, mediante las cuales tejen relaciones comunitarias y de cuidado y defensa del territorio, relaciones históricas que constantemente las comunidades indígenas, a través de su organización, propugnan por proteger, preservar y transmitir a las nuevas generaciones. 
En este sentido, es importante reconocer que iniciativas como esta han sido totalmente diferentes a los programas de reincorporación establecidos por el Gobierno Nacional y, sumado a esto, a lo planteado en los Acuerdos de Paz y la ruta concertada con la Agencia Colombiana de Reincorporación. Las principales diferencias que se evidencian entre la perspectiva institucional y la propuesta desde las comunidades se enmarcan en, primero, que desde la institucionalidad no se tienen en cuenta las formas de vida de las comunidades ni sus necesidades; segundo, el desconocimiento de la autonomía del derecho propio y la autoridad tradicional y su papel dentro del proceso de reincorporación (Verdad Abierta, 2015), y, tercero, las tendencias a la cooptación de la autonomía de las comunidades y sus procesos por parte de la institucionalidad.

\section{Acuerdo de paz, plan marco de implementación y avances a la implementación}

ruta para la construcción de una ruta de armonización que permitiera la reintegración e reincorporación de excombatientes indígenas quedó esbozada en el punto tres de los acuerdos, el correspondiente al "Fin del conflicto"; en este punto se plantearon a grandes rasgos las metas y objetivos para esta ruta, lo cual se desarrolló a profundidad en el Plan Marco de implementación.

Específicamente en este punto del acuerdo, se evidencia que en el Plan Marco de Implementación se acordó que "Se concertará con las organizaciones representativas de los pueblos étnicos un programa especial de armonización para la reincorporación de los desvinculados pertenecientes a dichos pueblos, que opten por regresar a sus comunidades, para garantizar el restablecimiento de la armonía territorial" (Departamento Nacional de Planeación, 2016, p.187). Para esto se planteó una meta específica a 2018, la cual correspondía a la construcción de un programa especial y con enfoque diferen- 
cial étnico y de género. Dicha meta se materializaba con el indicador que propendía por la construcción de un proceso de armonización que, bajo esta lógica, permitiera la reincorporación tanto económica como social de excombatientes. Dicha meta e indicador, si bien se concertaban con las comunidades de los Pueblos Indígenas, tenían una responsabilidad que recaía sobre la Agencia para la Reincorporación y la Normalización y que debía culminar en el año 2019; además, estaban enfocadas a la construcción de una política pública de reincorporación.

A la luz de estos elementos que fueron planteados para la construcción de esta ruta, en el año 2018 se hace oficial el CONPES 3931 del Departamento Nacional de Planeación, mediante el cual se presentaba la "Política Nacional para la reincorporación social y económica de exintegrantes de las FARC-EP” (CONPES 3931, 2018). En este se plantean los objetivos, enfoques, principios y se establece el plan de acción bajo 4 ejes fundamentales.

Inicialmente cabe resaltar que esta política cuenta con 4 enfoques, a saber: enfoque territorial, de derechos, de género y un enfoque étnico. El enfoque étnico "consiste en el conjunto de medidas y acciones que permiten dar un trato diferenciado a las personas que se auto reconocen como pertenecientes a un grupo étnico, en aras de garantizar el acceso a las oportunidades ofrecidas por el proceso de reincorporación en condiciones de equidad" (CONPES 3931 , p. 22); a su vez, este enfoque permite el restablecimiento de derechos de las familias de estos excombatientes y la comunidad indígena a la que pertenecen.

Para el ejercicio de investigación de este artículo, el enfoque territorial también es sumamente relevante, en esta política Nacional se define como aquel que busca "reconocer y tener en cuenta las necesidades, características y particularidades económicas, culturales y sociales de los territorios y las comunidades" (CONPES 3931, 
p. 32), a su vez, este enfoque busca que el desarrollo de la política cuente con la participación de las comunidades desde los territorios, reconociendo sus realidades, necesidades, autoridades regionales y actores relevantes dentro de la vida comunitaria.

En relación con esto, dentro de los objetivos planteados en esta política que da vida a la ruta de reincorporación con enfoque étnico, en su objetivo número dos se establece que se debe "Promover la reincorporación comunitaria en el territorio, orientada al fortalecimiento del tejido social, la convivencia y la reconciliación” (CONPES 3931, p. 72).

Este objetivo es fundamental, en cuanto el sentido comunitario y del territorio para el fortalecimiento del tejido social son elementos que ha dado vida a la construcción de iniciativas propuestas por el pueblo nasa, como el programa "Recomponer un camino de vuelta a casa”, mediante el cual como hemos mencionado previamente, pretende la rearmonización social y espiritual del excombatiente y la reconstrucción de sus lazos con la comunidad y el territorio. Es, en últimas, un esfuerzo por la reconstrucción del tejido social, que le apuesta a la construcción de paz territorial -en un sentido mucho más holístico- y la recuperación del sentido comunitario como elemento central para el Buen Vivir, reconociendo que se pretende recuperar en estos excombatientes la armonía comunitaria, sus costumbres y prácticas, y el respeto y defensa del territorio.

En este punto vale la pena plantear una enorme diferencia entre esta perspectiva que da vida a iniciativas propias de las comunidades y aquellas impulsadas desde la óptica institucional del Estado. Una de estas diferencias entre estos dos criterios -debate que desarrollaremos más adelante a profundidad- es que la construcción del tejido social para las comunidades es importante en tanto permite la recuperación de su vida comunitaria y sus lazos con el territorio; sin embargo, desde la perspectiva institucional -la cual se encuentra 
contenida en esta política- se pretende crear escenarios para la convivencia entre excombatientes y la comunidad a la que pertenecen, buscando así eliminar problemas relacionados con situaciones de estigmatización e intolerancia. Para esto, la política busca implementar estrategias, como la pedagogía para la paz, proyectos de trabajo conjunto entre excombatientes y las comunidades, proyectos productivos, entre otros, que chocan con la concepción de reincorporación de las comunidades -como los nasa-, y es que su concepción es también de armonización, es decir, un proceso que sana espiritualmente al sujeto y lo reconcilia con su comunidad y sus raíces.

En términos de implementación y avance, los indicadores que se trazan en el CONPES 3931 y en el Plan Marco de Implementación hasta la fecha no son significativos. En el Quinto informe referente a la verificación del estado de implementación del Acuerdo de paz, realizado por la Secretaria Técnica del CINEP (Centro de Investigación y Educación Popular) -periodo noviembre 2018 a enero de 2019-, se señalan que bajo el gobierno actual se intentaron agregar al Plan Nacional de Desarrollo las metas y los indicadores del Plan Marco de implementación correspondientes a la construcción del plan para la reintegración y reincorporación con enfoque diferencial étnico y de género, esto se realizó a través del "Pacto por la Equidad de oportunidades para Grupos indígenas, Negros, Afros, Raizales, Palanqueros y Rom". En este informe, presentado por el CINEP, se señaló que "las metas están en proceso de construcción entre el Gobierno y los grupos étnicos, así como también la articulación de éstas con el Plan Marco de Implementación” (Secretaría Técnica del Componente Internacional de Verificación, 2019a, p. 250).

En el Sexto informe referente a la verificación del estado de implementación, presentado también por el CINEP - periodo febrero a abril 2019-, se señala que hubo avances importantes en tanto "Fue aprobado el primer proyecto productivo colectivo con enfoque étnico, que beneficiará a 240 excombatientes, en su mayoría indígenas y 
que incluye un acuerdo de uso de la tierra con el resguardo indígena de Tumburao en Silvia, Cauca" (Secretaría Técnica del Componente Internacional de Verificación, 2019b, p. 109), también se destacó que

De acuerdo con el Registro Nacional de Reincorporación, 1.081 excombatientes hacen parte de grupos étnicos para lo cual la Agencia para la Reincorporación y la Normalización (ARN) junto con autoridades locales iniciarán la implementación del "Programa Especial de Armonización para reincorporación con enfoque étnico”. (Secretaría Técnica del Componente Internacional de Verificación, 2019, p. 110).

A través de la Agencia para la Reincorporación y la Normalización (ARN), en un derecho de petición contestado en 2018, se señaló que durante el periodo 2018-2019 se encontraban realizando la concertación y consulta con autoridades de los pueblos étnicos para la formulación del "Programa especial de armonización y reincorporación social y económica con enfoque diferencial étnico y de género". Para esto la ARN adelantó estrategias de apoyo técnico a entidades del Estado, autoridades internas y organizaciones de los pueblos étnicos, en cuanto a "procesos de diagnóstico, formulación, concertación y consulta" (Agencia para la Reincorporación y la Normalización, 2018); respecto al proyecto, también señaló que se avanzaba en la construcción conjunta de un borrador en el cual se adoptaría y reglamentaria el programa.

Finalmente, en el Sistema Integrado de Información para el Posconflicto (SIIPO), creado por la Alta Consejería para el Posconflicto y el Departamento Nacional de Planeación, los avances no son significativos. Dentro de los avances agrupados por puntos, según el Plan Marco de implementación presentados en el sistema con corte a $\mathbf{3 0}$ de junio de 2020, los 100 indicadores correspondientes al enfoque étnico solo presentan un avance del $12,05 \%$. A su vez, en el punto 3 del acuerdo (Fin del conflicto) se presenta un avance del 44,02\%. 
En cuanto a los 9 indicadores del enfoque étnico correspondientes al punto 3 la situación es preocupante (ver Imagen 1).

Étrico 喅
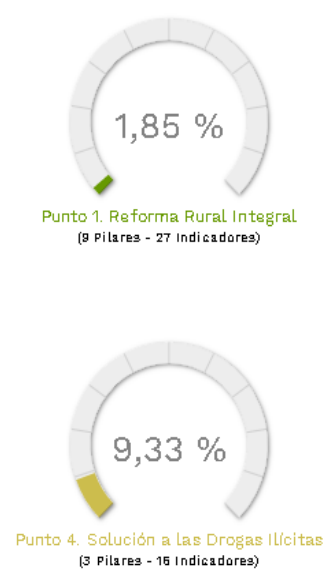
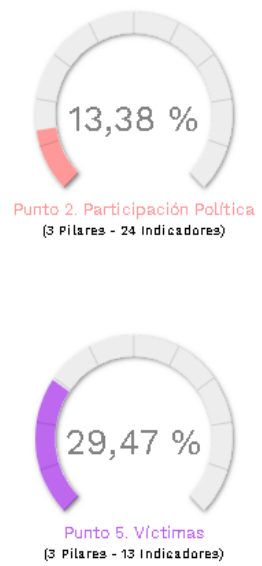

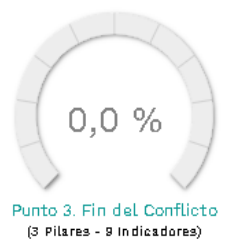

$27,27 \%$

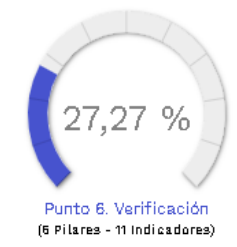

Imagen 1. Fuente: Sistema Integrado de Información para el Posconflicto (SIIPO)

Según la imagen anterior, con los datos presentado en el SIIPO los avances son del 0,0 \% aunque se reconoce que el "Programa especial de armonización para la reincorporación social y económica con enfoque diferencial étnico y de género" se encuentra concertado, diseñado y en estado de implementación, información que puede cruzarse con los avances del informe previamente presentado por la ARN.

\section{Aproximaciones a la noción de paz territorial y Buen Vivir}

El concepto de paz territorial tiene importantes exploraciones académicas previas. Para el ejercicio de esta investigación desarrollaremos un análisis comparado entre lo que teóricamente significa el Buen Vivir, con los planteamientos de Eduardo Gudynas (2011) y Alberto Acosta (2010), y la paz territorial, tomando los 
planteamientos de la profesora Carolina Jiménez Martín (2016) y Carolina Bautista (2017), así como dos perspectivas: la institucional, la cual analizaremos desde algunos planteamientos presentadas por funcionarios públicos, y una visión desde las comunidades, para las cuales tomaremos los planteamientos de Vilma Almendra, mujer nasa (Paez) quien en el libro Entre la emancipación y la captura: memorias y caminos desde la lucha Nasa en Colombia (2017), realiza reflexiones fundamentales.

Uno de los elementos más importantes que debemos tener en cuenta en principio es que se hace profundamente relevante analizar la construcción de territorialidades alternas a la concepción que se ha institucionalizado a través del Estado, bajo una dinámica neoliberal, que se reduce a funciones administrativas que justifican su explotación económica y el constante saqueo de los recursos económicos, lo que ha condenado a quienes habitan y defienden los territorios a condiciones de marginalidad y pobreza.

Inicialmente, el concepto de Buen Vivir, presentado por Eduardo Gudynas (2011), hace referencia a "un conjunto de ideas que se están forjando como reacción y alternativa a los conceptos convencionales sobre el desarrollo" (p. 1); en este sentido, es una concepción que pretende romper con las lógicas hegemónicas de un modelo neoliberal que limita la noción de desarrollo a una concepción económica y materialista.

Este conjunto de ideas se construye sobre la base, como menciona Alberto Acosta (2010), de "recuperar la cosmovisión de los pueblos y nacionalidades indígenas” (p. 11), convirtiéndose así en una visión mucho más holística de las condiciones humanas que permiten la configuración de buenos vivires. Para el ejercicio de este artículo, el cual pretende partir desde la noción de las comunidades estudiadas, el Buen vivir o la construcción de buenos vivires implica la construcción de relaciones armónicas entre los individuos de una comunidad y 
con ellos mismos, como también de relaciones armónicas con el territorio que habitan y la naturaleza; a su vez, implica la superación de la idea sobre la cual las condiciones de bienestar están enmarcadas en un modelo de vida impuesto de forma dominante enraizado en el consumismo, saqueo, acumulación y capacidad adquisitiva, propias del neoliberalismo y de la idea occidental de desarrollo.

Una vez desarrollado la noción de Buen Vivir, se hace relevante profundizar en lo que implica académicamente la noción de paz territorial. Cabe resaltar que en Colombia ha sido un concepto que se ha ido posicionando un el debate público posterior a la firma de los Acuerdos de Paz, pero que, al día de hoy, ha sido captado poco a poco por la institucionalidad. Sin embargo, esta discusión precisamente se aborda en el desarrollo académico que realizan las autoras que hemos mencionado previamente para abordar este tema, que en cierta forma logra configurarse como un concepto en disputa entre la parte institucional y una parte que le apuesta a la visión desde las bases sociales o comunitarias, es decir, se configura como un concepto en disputa que aún no se ha podido resolver al contar con horizontes de sentido diferentes.

Dentro de las experiencias académicas previas, la profesora Carolina Jiménez Martín comienza su aproximación al concepto de paz territorial reconociendo que "un tipo de paz territorial es la que propone la institucionalidad y las clases dominantes y otra a la le apunta el movimiento social y las clases populares" (Martín, 2016, p. 64). En este sentido, la perspectiva institucional ha propendido por posicionar la idea de la paz territorial como una estrategia para la transformación territorial enmarcada en una lógica desarrollista que se ha visto impedida por las dinámicas del conflicto. A su vez, esta estrategia implica un "trabajo articulado" entre la institucionalidad del Estado y las comunidades, dando paso a prácticas y normas de regulación territorial o nuevas formas de "gobernanza" funcionales a un modelo de desarrollo impuesto. 
Ante esto, Carolina Jiménez Martín manifiesta que la paz territorial implica un ejercicio de derechos, que pasa por reconocer las constantes "injusticias" territoriales que se agudizan y reproducen en el marco del conflicto como resultado a un orden social vigente. Asimismo, implica el reconocimiento de "horizontes de sentido diversos sobre el uso y formas de organizar el territorio" (Martín, 2016, p. 66), superando las condiciones de saqueo, acumulación y despojo que dan vida a un modelo económico materializado en el Estado y dando paso a territorialidades que se tejen desde lo comunitario. Esto permitiría entender que la "política transformativa de estas construcciones territoriales comunitarias radica en la posibilidad de usar y vivir el territorio desde una 'territorialidad comunitaria no saqueadora” (Martín, 2016, p. 64).

Para continuar con este planteamiento, la profesora Carolina Bautista evidencia la importancia de reconocer la paz desde una perspectiva territorial, ya que se hace relevante reconocer que la configuración del territorio implica unas dinámicas sociales que lo configura, pero que, a su vez, generan lógicas de apropiación y construyen territorialidades. Bajo esta lógica, la paz territorial implica permitir que se construya en concordancia con las demandas, necesidades y agendas de los territorios, desde la perspectiva de quienes lo habitan, por y para ellos. Frente a esto, Carolina Bautista menciona que implica "centrar la mirada y edificar la paz territorial desde los procesos de convivencia comunitaria” (Bautista, 2017, p. 106).

La construcción de territorialidades en experiencias, como la de los nasa, en el programa "Recomponer un camino de vuelta a casa", que le apuestan a la construcción de paz, son fundamentales en tanto que es evidente que responden a las necesidades, lógicas, demanda y agenda de su comunidad y a un sentido de pertenencia producto de sus relaciones culturales, políticas y sociales que, además, tejen alrededor de sus territorios históricos. Cuando planteamos la división con la lógica institucional, poco a poco logramos evidenciar 
que para estas comunidades el territorio no es una mera división geográfica, sino más bien una conexión de vida, donde hay una pugna constante por defender sus concepciones vitales sobre este y su importancia para la vida de su comunidad.

Al respecto Vilma Almendra comienza sosteniendo que, si bien el proceso de paz abrió la puerta para la discusión de temas relevantes, la realidad es que en los territorios las comunidades continúan -y continuarán- bajo dinámicas históricas de saqueo, despojo, guerra, etc., producto claramente del modelo neoliberal bajo el cual se encuentra nuestro país y, además, modelo al cual los acuerdos son profundamente funcionales. Reconoce que con los acuerdos se pretendió, por un lado, el desarme de las FARC-EP por parte del gobierno y, por otro, poder asegurar la entrada y desarrollo de iniciativas económicas de capitales trasnacionales. Referente a esto, una de las reflexiones presentadas por la autora es:

La cooptación-captura-contención estatal se está manifestando a tal punto que hasta algunas comunidades y concretamente luchadores y luchadoras históricas del movimiento indígena y popular, han sentado su voz de protesta frente a una propuesta de paz condicionada a la reproducción del capital, con políticas mineroenergéticas que profundizan las condiciones sociales que provocaron el conflicto armado, pero también intentan diezmar la lucha anticapitalista, porque de entrada se pretende institucionalizar cualquier amenaza subversiva y se están encadenando a esa paz, las luchas, resistencias y autonomías que constituyen la real posibilidad de una paz indígena, negra, campesina y popular para emanciparse. (Almendra, 2017, p. 207)

Dicha captura estatal a la cual se hace referencia, inicialmente puede entenderse de tres formas: 1) una captura organizativa en tanto se evidencian intenciones por parte del Estado de acercar líderes y lideresas a través de beneficios para entrar a negociar acuerdos sobre los territorios; 2) una captura política si hay un intención de varios actores -entre ellos el Estado- de apropiarse de narrativas y discursos que las comunidades han tejido y construido 
desde sus experiencias vitales, con el objetivo de apropiarse de los derechos adquiridos a través de estos y continuar subordinando a las comunidades que los construyeron; 3) una captura semántica, mientras también se genera una apropiación de sus concepciones y prácticas, que han surgido para nombrar sus necesidades, prácticas, crisis y alternativas.

La reflexión anteriormente planteada no se hace ajena a los acuerdos de paz. Como se mencionó anteriormente, los acuerdos han sido funcionales a los intereses del Estado neoliberal, al cual, bajo ninguna circunstancia, le favorece reconocer la autonomía de las comunidades, de sus planes y proyectos de vida que están encaminados a la emancipación y transformación comunitaria. Valdría la pena analizar o dejar para la reflexión si desde el Estado se cuenta con la capacidad de erradicar dicha autonomía, por tanto, su única alternativa es generar procesos de cooptación y captura.

Haciendo un paréntesis, a esto se le puede sumar que hubo una tendencia para que primara lo económico dentro de la agenda de paz negociada en los acuerdos. Hay que reconocer que varias visiones apuntaban a que el origen del conflicto se centraba en que los principales síntomas de la guerra en Colombia partían de causas como la pobreza, la desigualdad en la distribución del ingreso, etc. Esta visión que es sustentada, por ejemplo, por Carlo Nasi (2003), quien sostiene que "la guerra en Colombia es esperable dado que cerca del $20 \%$ de los habitantes viven en condiciones de pobreza absoluta, y el país padece una de las peores distribuciones de ingreso en el continente, medida por el índice de Gini” (p. 89) por lo cual se hacía profundamente importante incluirlos como punto de partida para una posible agenda de negociaciones de paz entre el gobierno y grupos insurgentes, desconociendo otro tipo de condiciones de carácter político y social que también dieron origen al conflicto. 
Continuando con la discusión referente al carácter funcional del acuerdo y la cooptación institucional del mismo y de los procesos comunitarios, a esto el Ex alto comisionado para la paz, Sergio Jaramillo, en una presentación sobre los acuerdos en la Universidad de Harvard, sostuvo que los acuerdos eran una oportunidad para fortalecer la institucionalidad en los territorios, agregó que debía hacerse de forma conjunta y "desde abajo" entre las comunidades y el Estado. Sin embargo, indicó que:

(...) tampoco se trata de que las comunidades se organicen por su cuenta. Esa es una lógica de fragmentación, que ha sido nuestra enfermedad histórica. Lo que necesitamos es imponer una lógica de inclusión e integración territorial, basada en una nueva alianza entre el Estado y las comunidades para construir conjuntamente institucionalidad en el territorio. 'Institucionalidad' entendida nuevamente no sólo como la presencia de unas entidades estatales, sino como el establecimiento conjunto de unas prácticas y normas que regulen la vida pública y produzcan bienestar. (Jaramillo, 2014)

La evidente funcionalidad de los acuerdos de paz a dinámicas de explotación, saqueo y despojo, propias del sistema neoliberal, se evidencian en algunas declaraciones que rodearon el proceso de negociación, al respecto Juan Carlos Echeverri (expresidente de Ecopetrol) manifestó en una entrevista al Espectador, en el año 2016, que:

Con la paz esperaríamos tener [sic] la posibilidad de entrar a Caquetá mucho más fuerte, a Putumayo, a Catatumbo, sitios donde antes era difícil acceder. La paz nos debe permitir no solamente a Ecopetrol, sino a todas las petroleras del país, generando desarrollo en las regiones, sacar más petróleo. (Echeverri, 2016)

Estas afirmaciones son analizadas también por la profesora Carolina Bautista; menciona que es una clara intención por "llenar un vacío e institucionalizar el territorio" (Bautista, 2017, p.104), vacío que en este tipo de discursos hace referencia a una precaria $-\mathrm{O}$ nula- presencia del Estado. A través de estas afirmaciones realizadas, 
personajes como el alto comisionado para la paz pretenden desconocer otras formas mediante las cuales se tejen interacciones sociales, políticas sociales, apuestas organizativas en los territorios, y demuestra "el claro propósito de disputar las construcciones sociales y políticas existentes al margen del Estado centralista" (Bautista, 2017, p. 104) o de la institucionalidad "formal".

Podría entenderse que "la paz permitida desde la institucionalidad global no es otra cosa que una nueva cadena, atadura y cerco que pretende limitar nuestras reales apuestas, prácticas y luchas cotidianas y de largo aliento" (Almendra, 2017, p. 212-213). Esta misma discusión es la que impulsa a la creación de programas como el que ha impulsado esta investigación, que desde las comunidades le apuestan a la superación del conflicto social, político y económico, de forma estructural, a través de su autonomía y de acuerdo a sus necesidades, prácticas, disputas, etc.

Esto no quiere decir que las comunidades no aprueben los acuerdos ni el proceso. $\mathrm{Al}$ respecto Vilma menciona que:

(...) nosotros esperamos que la implementación de dicho acuerdo se realice de la mejor manera posible estableciendo el cese al fuego definitivo y permanente entre las partes, inicialmente con las FARC y ahora con el Ejército de Liberación Nacional (ELN), para que bien desde abajo y con tranquilidad en nuestros territorios podamos seguir gestando las apuestas y prácticas de paz que históricamente venimos caminando. (Almendra, 2017, p. 216)

Es menester reconocer que las comunidades muchas veces crean procesos de resistencia frente a la cooptación institucional del Estado, impulsados por la defensa de sus saberes, formas de vida, perspectivas y necesidades, pero también desde la forma como el conflicto afectó e impactó sus prácticas y planes de vida. El reconocimiento de estas visiones y choques irreconciliables bajo la lógica del Estado neoliberal se hace evidente. Frente a esto Vilma menciona que: 
Ellas y ellos quienes le ponen el pecho a la guerra día a día en sus territorios, nos enseñan que nuestra manera otra de vivir plenamente coexistiendo con todos los seres de la naturaleza y de alimentar nuestras formas otras de hacer política desde la comunidad, son ya una práctica concreta que se opone a la muerte que impone el capital, por lo tanto, constituye nuestra apuesta por la paz. (Almendra, 2017, p. 216)

En específico, la conformación de un proceso diferenciado y que permita la recuperación y rearmonización de miembros de sus comunidades es una apuesta por ello. La superación del conflicto y la creación de escenarios de paz en sus territorios son apuestas por el Buen Vivir que buscan, también, la vida en armonía de las comunidades y, a su vez, tejer relaciones de cuidado y respeto entre estas, así como con la tierra, el territorio y los otros seres que lo habitan.

\section{Conclusiones}

1. Para responder a la pregunta que motivó esta investigación, el programa de armonización propuesto por el pueblo nasa logra configurarse como una oportunidad para la construcción de paz territorial y buen vivir porque está construido bajo una visión holística y una perspectiva emancipadora que le apuesta, desde la autonomía, a condiciones que garanticen su permanencia y defensa del territorio; asimismo, está fundamentado en la recuperación de las relaciones de armonía y de respeto con y hacia la naturaleza, el territorio y quienes lo habitan. Por último, es un esfuerzo para responder a la cooptación institucional que pretende inviabilizar y desconocer a quienes, a través de este tipo de iniciativas, han construido paz en los territorios desde su autonomía, saberes, sentidos y formas de vida que rompen con la lógica desarrollista de la paz.

2. Continuar con la discusión sobre lo que es e implica la construcción de paz territorial es necesario, esto 
reconociendo que tiene visiones y perspectivas que no debemos desconocer en cuanto son enriquecedoras para el debate académico, político y social.

3. Si bien los acuerdos de paz cuentan con un potencial transformador que pretendía desmontar algunas condiciones que sostuvieron y reprodujeron el conflicto, desde las perspectivas de los pueblos indígenas, como los Nasa, se hace inviable mientras representa una visión y modelo de Estado que no es congruente con sus formas de vida. A esto se le suman los precarios niveles de implementación, sobre todo en la política de reincorporación con enfoque étnico que favorezca a estas comunidades y esté construido desde sus lógicas, visiones y formas de vida.

4. Es imposible saldar por consenso el debate de lo que implica la construcción de paz territorial entre la comunidad académica, las comunidades indígenas y la perspectiva institucional. La naturaleza misma del Estado neoliberal lo imposibilita al estar llena de contradicciones y condiciones materiales opuestas a las planteadas en las formas de vida de los pueblos indígenas como los Nasa. Esta discusión tampoco se salda con la implementación de una política de rearmonización con enfoque étnico propuesta desde la visión institucional, ya que desconoce que para las comunidades indígenas el proceso de reincorporación no se da solo en términos sociales y económicos, sino también en términos espirituales y comunitarios, elementos que no son relevantes para el Estado.

5. Es fundamental que como sociedad aprendamos de las experiencias y propuestas enriquecedoras y necesarias que nacen desde las comunidades, como lo es el programa "Recomponer un camino de vuelta a casa", ya que sin duda es una enseñanza significativa para quienes, si bien reconocemos el potencial de los acuerdos, no podemos 
dejar de lado su funcionalidad, es decir, un sistema que ha condenado a nuestros pueblos y sus territorios a condiciones de explotación, miseria y muerte. Esto implica respetar estas iniciativas y la autonomía de los pueblos indígenas, así como entender que, probablemente, su organización y lucha propenderán por resistir a las lógicas hegemonizantes del Estado neoliberal, y que el acuerdo de paz y un programa de reincorporación con enfoque étnico institucionalizado no será la excepción.

\section{Referencias}

Acosta, A. (2010). El Buen Vivir en el camino del post-desarrollo: Una lectura desde la constitución de Montecristi.

Agencia para la Reicorporación y Normalización. (2018). CONPES 3931.

Agencia para la Reincorporación y la Normalización. (2018). Respuesta derecho de petición. Bogotá.

Agencia para la Reincorporación y Normalización. (03 de 07 de 2018). camara.gov. vo. Obtenido de https://www.camara.gov.co/sites/default/files/2020-05/RESPUESTA\%20AGENCIA\%20PARA\%20LA\%20REINCORPORACI\%C3\%93N\%20 Y\%20NORMALIZACI\%C3\%93N.pdf

Almendra, V. (2017). Entre la emancipación y la captura: memorias y caminos desde la lucha Nasa en Colombia. México: Barricadas Colecciones.

Alto Comisionado para la Paz Gobierno de Colombia. (2016). ACUERDO FINAL PARA LA TERMINACIÓN DEL CONFLICTO Y LA CONSTRUCCIÓN DE UNA PAZ ESTABLE Y DURADERA . ACUERDO FINAL PARA LA TERMINACIÓN DEL CONFLICTO Y LA CONSTRUCCIÓN DE UNA PAZ ESTABLE Y DURADERA .

Bautista, C. (2017). Contribuciones a la fundamentación conceptual de paz territorial. Ciudad Pasando.

DANE. (2005). Censo General 2005. Bogotá.

DANE. (2018). Censo Nacional de Población y Vivienda 2018. Bogotá. 
Departamento Nacional de Planeación. (2016). Plan marco de Implementación.

Echeverri, J. C. (14 de Abril de 2016). "La paz nos va a permitir sacar más petróleo de zonas vedadas por el conflicto". (E. Espectador, Entrevistador)

Estrada, J. (2019). El acuerdo de paz en Colombia: entre la perfidia y la potencia transformadora. En J. Estrada, El acuerdo de paz en Colombia: entre la perfidia y la potencia transformadora (p. 23). Bogotá: CLACSO.

Gudynas, E. (2011). Buen Vivir: germinando alternativas al desarrollo. América Latina en movimiento, (462), 1-20.

Martín, C. J. (2016). Justicia territorial para la construcción de paz. Bictacora, 26.

Miller A. (2020). ).¿Por qué continúa la guerra en el Cauca? Bogotá: Comisión de la verdad.

Nasi, C. (2003). Agenda de paz y reformas: ¿Qué se puede y qué se debe negociar? Revista de estudios sociales, 14, 88-105. https://revistas.uniandes.edu.co/doi/abs/10.7440/ res14.2003.08

Procuraduria General de la Nación. (2019). Pueblo indígena Nasa Caracterización.

Secretaría Técnica del Componente Internacional de Verificación. (2019a). Quinto informe de verificación de la implementación del Acuerdo Final de Paz en Colombia para los Verificadores Internacionales. Centro de Investigación y Educación Popular (CINEP) y el Centro de Recursos para el Análisis de Conflictos (CERAC).

Secretaría Técnica del Componente Internacional de Verificación. (2019b). Sexto informe de verificación de la implementación del Acuerdo. Centro de Investigación y Educación Popular (CINEP) y el Centro de Recursos para el Análisis de Conflictos (CERAC).

Sergio Jaramillo. (2014, 13 de marzo). La Paz Territorial [conferencia]. Universidad de Harvard, Cambridge, UU. EE.

Universidad Nacional de Colombia. (2017). Caracterización comunidad FARC-EP. Universidad Nacional de Colombia.

Verdad Abierta. (2015). El regreso a casa de los indígenas NASA que pasaron por la guerra. Verdad Abierta. 